Research Article

\title{
Elevation of Platelet and Monocyte Activity Markers of Atherosclerosis in Haemodialysis Patients Compared to Peritoneal Dialysis Patients
}

\author{
Ksenija Stach, ${ }^{1}$ Susanne Karb, ${ }^{1}$ Ibrahim Akin, ${ }^{1}$ Martin Borggrefe, ${ }^{1}$ Bernhard Krämer, ${ }^{2}$ \\ Thorsten Kälsch, ${ }^{1,3}$ and Anna-Isabelle Kälsch ${ }^{2}$ \\ ${ }^{1} 1$ st Medical Department, University Medical Centre Mannheim, Medical Faculty Mannheim, University of Heidelberg, \\ Heidelberg, Germany \\ ${ }^{2} 5$ th Medical Department, University Medical Centre Mannheim, Medical Faculty Mannheim, University of Heidelberg, \\ Heidelberg, Germany \\ ${ }^{3}$ Herzzentrum Weinheim, University Medical Centre Mannheim, Medical Faculty Mannheim, University of Heidelberg, \\ Heidelberg, Germany
}

Correspondence should be addressed to Anna-Isabelle Kälsch; Anna-isabelle.kaelsch@umm.de

Received 24 January 2017; Revised 29 May 2017; Accepted 11 June 2017; Published 9 July 2017

Academic Editor: Giuseppe Valacchi

Copyright ( 92017 Ksenija Stach et al. This is an open access article distributed under the Creative Commons Attribution License, which permits unrestricted use, distribution, and reproduction in any medium, provided the original work is properly cited.

Purpose. The predominant cause of mortality in dialysis patients are cardiovascular events. Platelet and monocyte activity markers play an important role in cardiovascular mortality and were assessed and related to dialysis quality criteria in haemodialysis (HD) and peritoneal dialysis (PD) patients. Methods. For this prospective comparative study, HD patients $(n=41)$ and PD patients $(n=10)$ were included. In whole blood samples, surface expression of CD62P and CD40L on platelets, tissue factor binding on monocytes, and platelet-monocyte aggregates were measured by flow cytometry. Plasma levels of MCP-1, IL-6, TNF $\alpha$, and soluble CD40L were analysed by enzyme-linked immunosorbent assay. Results. Haemodialysis patients showed a significantly higher CD62P expression on platelets $(p=0.017)$, significantly higher amount of platelet-monocyte aggregates $(p<0.0001)$, and significantly more tissue factor binding on monocytes $(p<0.0001)$ compared to PD patients. In PD patients, a significant correlation between Kt/V and platelet CD40L expression $(r=0.867 ; 0.001)$ and between $\mathrm{Kt} / \mathrm{V}$ and platelet CD62P expression $(r=0.686 ; p=0.028)$ was observed, while there was no significant correlation between $\mathrm{Kt} / \mathrm{V}$ and tissue factor binding on monocytes and platelet-monocyte aggregates, respectively. Conclusion. Platelet and monocyte activity markers are higher in HD patients in comparison with those in PD patients, possibly suggesting a higher risk of cardiovascular morbidity and mortality.

\section{Introduction}

End-stage renal disease is associated with increased cardiovascular mortality and morbidity. The mortality rate in patients with end-stage renal disease (ESRD) is comparable to many cancers and is mostly caused by an increased mortality rate due to cardiovascular disease. A promising approach to reduce this mortality has been to increase dialysis dose. Therefore, possible correlations between dialysis dose and mortality have been studied in major trials with disappointingly negative results for both haemodialysis and peritoneal dialysis patients [1,2]. Furthermore, increased circulating inflammatory proteins have been shown to predict a worse prognosis in ESRD patients [3].

Alterations in haemostasis are common complications of kidney diseases. Their frequency and severity correlate with the progressive loss of renal function to end-stage renal disease. Both bleeding diathesis and thromboembolic events 
have been identified [4]. The coagulation profile of patients with ESRD shows normal or elevated levels of coagulation factors, suggesting that occurrence of haemorrhagic events is mainly due to platelet dysfunction [5]. Proper haemostasis requires adhesion and aggregation of platelets at the site of vascular injury, as well as secretion of several adhesive substances by platelets [6]. The altered platelet function in ESRD is the result of impaired platelet adhesiveness as well as abnormal platelet endothelial interaction [7]. An interaction between activated platelets and endothelial cells plays an important pathophysiologic role in the development of atherosclerosis [8]. In detail, interactions between platelets and endothelial cells mediate essential processes in the development of atherosclerosis by an increased expression of vascular cell adhesion molecules and their ligands. These include P-selectin-an $\alpha$-granule protein that mediates platelet rolling, leukocyte adhesion, and coagulation-tissue factor expression on monocytes, platelet-monocyte aggregates, and CD40L-a member of the tumor necrosis factor- $\alpha$ family of proteins [9]. CD62P and CD40L are expressed on activated platelets and are directly involved in the interaction of platelets with monocytes and endothelial cells [10].

The predominant cause of the mortality of dialysed patients are cardiovascular events related to thrombosis [11]. In this respect, the CD40 receptor and its ligand (CD40L) on activated platelets are of particular interest. They are known to modulate both inflammation and thrombosis, two processes important for the development and clinical expression of atherosclerosis [12]. Binding of CD40L to its CD40 receptor on endothelial cell membranes induces an enhanced release of potent proinflammatory and atherosclerosis-promoting cytokines and chemokines (e.g., IL-6 and MCP-1) [13]. Tissue factor expression on monocytes is important in extrinsic coagulation and elevated in HD patients compared to that in healthy controls [14]. It is known that a disturbed platelet function is an important cause for thromboembolic and haemorrhagic complications in ESRD patients.

Haemodialysis and peritoneal dialysis are both established methods in treating ESRD and aim to attenuate cardiovascular risk factors. In order to investigate possible functional differences between both dialysis modalities regarding and affecting atherothrombotic risk, the present study assessed platelet and monocyte activity markers of atherosclerosis and correlated these markers to dialysis dose in both dialysis modalities.

\section{Methods}

2.1. Study Population. For this prospective comparative study, haemodialysis patients ( $n=41 ; 25$ male, 16 female) or peritoneal dialysis patients ( $n=10 ; 6$ male, 4 female) were included consecutively.

The investigation conforms to the principles outlined in the Declaration of Helsinki. The study was approved by the local ethics committee and all patients gave informed consent to study participation and the use of their medical record for research purposes.
Haemodialysis patients were haemodialysed on hollowfiber polysulfone membrane dialysators with a $1.3 \mathrm{~m}^{2}$ surface area $(n=40)$ (Polysulfone UF 5.5; Hemoflow F6, Fresenius, Germany) or with cellulose triacetate dialysator $(n=1)$ (CT 150 G, Baxter, Germany) with bicarbonate-containing solutions for on average 13.2 (9-20) hours weekly. Peritoneal dialysis solutions of 21 with $1.36 \%$, and when necessary $3.86 \%$, glucose were used three or four times a day by the patients using peritoneal dialysis. All the patients were advised to comply with a chronic renal failure diet consisting of $35 \mathrm{kcal} / \mathrm{kg}$, including 1.2/1.4 g/kg of protein, 1000/1500 mg of calcium, $700 \mathrm{mg}$ of phosphorus, and $250 \mathrm{mg}$ of magnesium, vitamins, and recombinant human erythropoietin. The $\mathrm{Kt} / \mathrm{V}$ was $1.53 \pm 0.28$ for hemodialysis patients and $2.63 \pm 0.65$ for peritoneal dialysis patients.

2.2. Flow Cytometric Analysis. Blood samples of heparinized blood (15 IE heparin per ml blood) (Sarstedt AG \& Co., Nuembrecht, Germany) were obtained. To avoid aggregation and activation of platelets, the blood samples were shaken over the time. All whole blood samples were subsequently investigated on activation of monocytes and platelets as well as platelets binding on monocytes.

Flow cytometric analysis of platelets was performed by gating in forward and side scatter. Platelets were gated back for determination of the expression of CD40L and CD62P. Platelet-monocyte aggregates were measured by CD41 (GPIIb/IIIa receptor) surface expression on platelets adherent to monocytes.

For the analysis of platelets, $100 \mu \mathrm{l}$ of each whole blood sample was stained for $30 \mathrm{~min}$ at room temperature with $10 \mu \mathrm{l}$ aliquots of mouse anti-human CD62P-PE antibodies (CLB-Thromb/6) (Coulter Immunotech, Krefeld, Germany) and mouse anti-human CD40L-FITC antibodies (P2) (Calbiochem/Merck KGaA, Darmstadt, Germany). For the analysis of monocytes, $100 \mu$ l of each whole blood sample was stained for $30 \mathrm{~min}$ at room temperature with $10 \mu \mathrm{l}$ aliquots of PE-conjugated murine antibody against CD41 (Coulter Immunotech, Krefeld, Germany) and murine FITC-conjugated antibody against tissue factor (American Diagnostica, Pfungstadt, Germany). To identify monocytes, the probes were additionally stained with mouse antihuman CD14-ECD (RM052) (Coulter Immunotech, Krefeld, Germany). Isotype-matched mouse anti-human IgG1 PE/ FITC antibodies (Beckman Coulter, Marseille, France) were used as a control. After incubation, erythrocytes were lysed with $500 \mu$ l Optilyse C (Coulter Immunotech, Krefeld, Germany). After $15 \mathrm{~min}$, cells were resuspended in $500 \mu \mathrm{l}$ PBS and were then ready for flow cytometric analysis. For measurement of CD62P and CD40L on platelets, a gating for forward and sideward scatter was performed. For measurement of CD41 on platelets adherent to monocytes to determine platelet-monocyte aggregates and for measurement of membrane-bound tissue factor, a gating for the monocyte surface antigen CD14 and sideward scatter was performed. All flow cytometric analysis was performed on an EPICS XL-MCL analyzer (Beckman Coulter, Krefeld, Germany) equipped with an argon laser tuned at $488 \mathrm{~nm}$. Compensation of the four-channel fluorescence was precisely 
TABLE 1: Study population.

\begin{tabular}{|c|c|c|c|c|c|}
\hline & HD patients $n=41$ & HD patients (\%) & PD patients $n=10$ & PD patients (\%) & $p$ value \\
\hline Age (years) & $61.83 \pm 15.42$ & & $53.2 \pm 11.25$ & & 0.126 \\
\hline Male & 25 & 60.98 & 6 & 60 & 1 \\
\hline Hyperlipidemia & 6 & 14.63 & 6 & 60 & 0.007 \\
\hline Coronary artery disease & 12 & 29.27 & 2 & 20 & 0.707 \\
\hline Peripheral arterial disease & 8 & 19.51 & 2 & 20 & 1 \\
\hline Diabetes mellitus & 15 & 36.59 & 1 & 10 & 0.142 \\
\hline Hypertension & 35 & 85.37 & 10 & 100 & 0.331 \\
\hline
\end{tabular}

TABLe 2: Medication in use at baseline.

\begin{tabular}{|c|c|c|c|c|c|}
\hline Medication & HD patients $n=41$ & HD patients (\%) & PD patients $n=10$ & PD patients $(\%)$ & $p$ value \\
\hline$\overline{\mathrm{ASA}}$ & 5 & 12.2 & 1 & 10 & 1 \\
\hline Oral anticoagulants & 9 & 21.95 & 1 & 10 & 0.664 \\
\hline Beta blocker & 22 & 53.66 & 5 & 50 & 1 \\
\hline Statins & 4 & 9.76 & 4 & 40 & 0.038 \\
\hline ACE-inhibitor/AT(1) antagonist & 20 & 48.78 & 6 & 60 & 0.727 \\
\hline
\end{tabular}

ASA = acetylsalicylic acid; ACE inhibitor = angiotensin-converting-enzyme inhibitor; AT (1) antagonist = angiotensin (1) antagonist.

adjusted using Cyto-CompTM reagents and Cyto-TrolTM control cells (Coulter Immunotech, Krefeld, Germany). We followed the methods of Pirzer et al. 2012 [15].

2.3. Enzyme-Linked Immunosorbent Assay (ELISA). Plasma concentrations of TNF $\alpha$ (Human TNF $\alpha /$ TNFSF1A Immunoassay R\&D Systems, Inc., Wiesbaden, Germany), interleukin 6 (IL-6) (Human IL-6 Immunoassay R\&D Systems, Inc., Wiesbaden, Germany), monocyte chemotactic protein-1 (MCP-1) (Human MCP-1/CCL2 Immunoassay R\&D Systems, Inc., Wiesbaden, Germany), and CD40L (Human soluble CD40 Ligand Immunoassay R\&D Systems, Inc., Wiesbaden, Germany) were determined by sandwich-type immunoassay according to the manufacturer instructions. All concentration analysis was performed on an ELISAReader-Lab Systems Multiskan RC (Lab Systems, Finland). Genesis Lite Software and ELISA Multiskan RC were used for data acquisition and evaluation.

2.4. Statistical Analysis. Numerical data were expressed as mean \pm standard deviation (SD). A Mann-Whitney test was applied as a nonparametric test. Categorical variables were analysed using a chi-square test and $t$-test. Values are expressed as mean values \pm SD. A two-tailed probability $<0.05$ was considered significant. All calculations were performed using GraphPad InStat version 3.01 (GraphPad Software, San Diego, California, USA) and SPSS Statistics version 17 (SPSS-Software GmbH, Munich, Germany).

\section{Results}

Baseline characteristics of the study patients as well as the medication use at baseline are given in Tables 1 and 2 .
3.1. Difference between Haemodialysis and Peritoneal Dialysis regarding Platelet and Monocyte Activity Markers. HD patients had significantly higher CD62P expression on platelets compared to $\mathrm{PD}$ patients $(1.9 \pm 0.9$ versus $1.6 \pm 1.5$; $p=0.017$ ) (Figure 1). We also found a significantly higher amount of platelet-monocyte aggregates (19.3 \pm 10.4 versus $6.6 \pm 4.2 ; p<0.0001$ ) (Figure 2) and tissue factor binding on monocytes $(1.3 \pm 0.3$ versus $0.9 \pm 0.2 ; p<0.0001)$ in $\mathrm{HD}$ patients compared to that in PD patients (Figure 3 ). There was no significant difference between both patient groups regarding platelet surface expression of CD40L.

There was no significant correlation between Kt/V and any of the determined parameters in HD patients (data not shown). In PD patients, we found a significant correlation between $\mathrm{Kt} / \mathrm{V}$ and CD40L $(r=0.867 ; 0.001)$ and between $\mathrm{Kt} / \mathrm{V}$ and CD62P $(r=0.686 ; p=0.028)$, while there was no significant correlation between $\mathrm{Kt} / \mathrm{V}$ and tissue factor binding on monocytes and platelet-monocyte aggregates, respectively (data not shown).

3.2. Correlation of Weekly Dialysis Duration with Expression of Tissue Factor. The expression of tissue factor on monocytes was significantly decreased in comparison to increasing duration of haemodialysis sessions $(p=0.031)$ (Figure 4$)$.

3.3. Effect between Haemodialysis and Peritoneal Dialysis on Cytokine Production. HD patients had significantly higher cytokine production of TNF $\alpha$ compared to PD patients $(9.25 \pm 11.13$ versus $3.56 \pm 1.24 ; p=0.019)$ (Figure 5). There were no significant differences between both patient groups regarding cytokine production of IL-6, MCP-1, and SCD40L. 


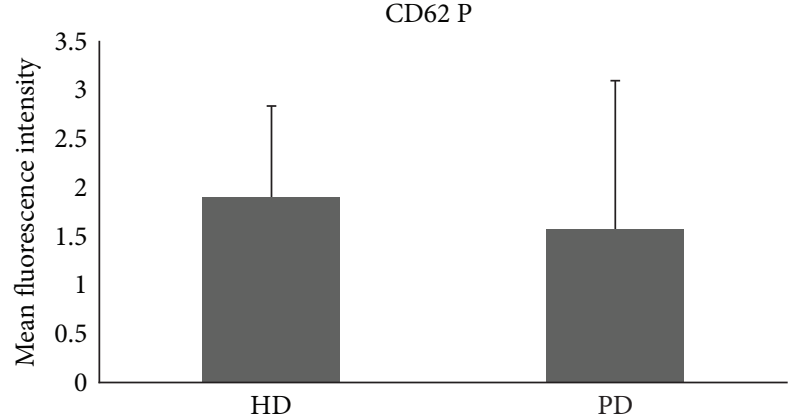

FIgure 1: Significant difference between HD and PD on surface expression of CD62 $\mathrm{P}$ on platelets $(p=0.017)$. HD = haemodialysis; $\mathrm{PD}=$ peritoneal dialysis.

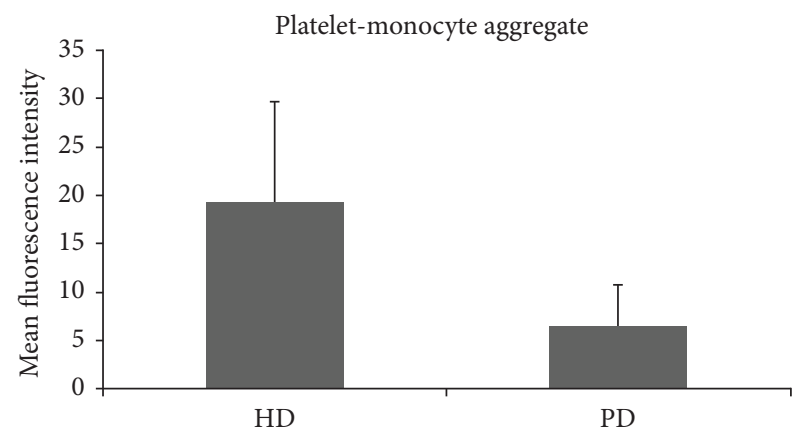

Figure 2: Significant difference between $\mathrm{HD}$ and $\mathrm{PD}$ on platelet-monocyte aggregates $(p<0.0001) . \mathrm{HD}=$ haemodialysis; $\mathrm{PD}=$ peritoneal dialysis.

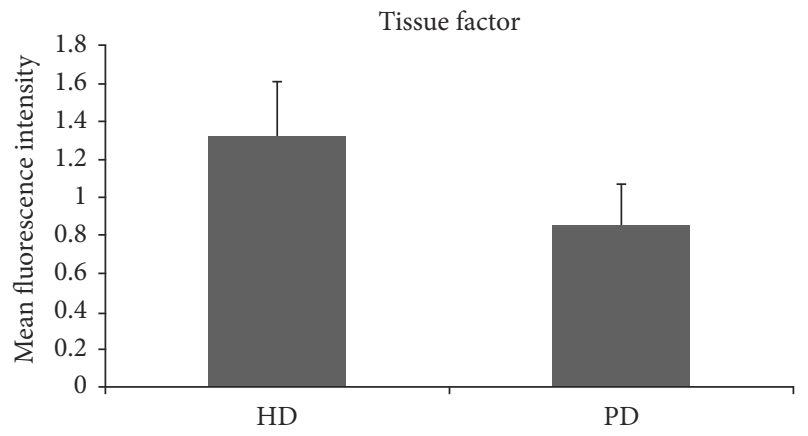

Figure 3: Significant difference between $\mathrm{HD}$ and $\mathrm{PD}$ on surface expression of tissue factor on monocytes $(p<0.0001)$. $\mathrm{HD}=$ haemodialysis; $\mathrm{PD}=$ peritoneal dialysis .

\section{Discussion}

Patients with end-stage renal disease (ESRD) undergo renal replacement therapy to avoid fatal complications of uremic toxins, elevated potassium level, and over hydration. Unfortunately, ESRD is conjoined with increased atherosclerotic and thromboembolic morbidity and mortality. In Germany, renal replacement therapy involves mostly haemodialysis and in a smaller proportion peritoneal dialysis treatments.

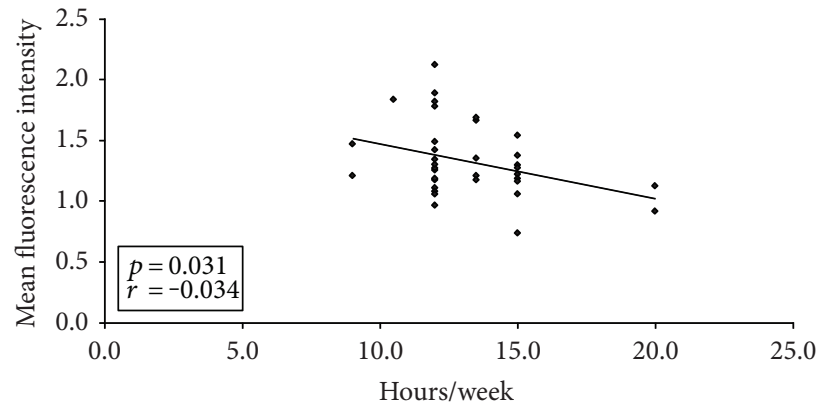

FIgURe 4: The expression of tissue factor on monocytes was significantly decreased in comparison to increasing time of haemodialysis $(p=0.031)$. indicates tissue factor.

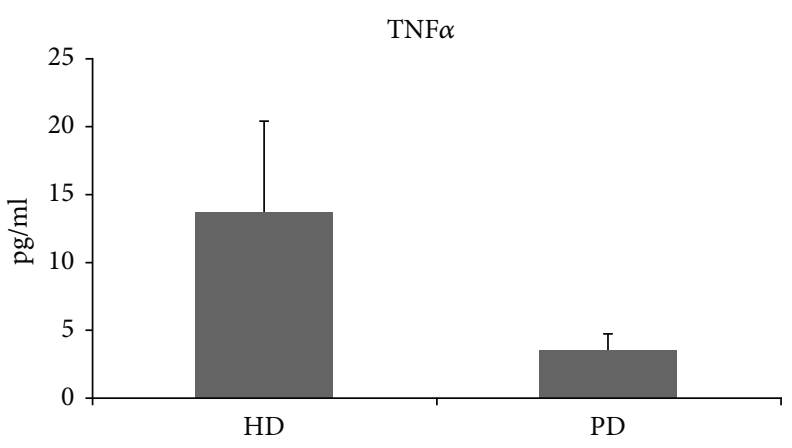

Figure 5: Significant difference between $\mathrm{HD}$ and PD on cytokine production of $\mathrm{TNF} \alpha(p=0.019) . \mathrm{HD}=$ haemodialysis; $\mathrm{PD}=$ peritoneal dialysis.

Therefore, the aim of this study was to investigate a possible difference between $\mathrm{HD}$ and $\mathrm{PD}$ with respect to the expression of platelet and monocyte activity markers and the tissue factor system. Numerous authors have previously addressed the question of platelet and coagulation activation in haemodialysis patients [16-18]. One important finding is that the presence of cardiovascular disease is closely linked to the tissue factor system [19]. Yorioka et al. described higher levels of tissue factor on monocytes in haemodialysis patients in comparison to a healthy control group [14]. Pawlak et al. demonstrated a relationship between increased oxidative stress and elevated tissue factor in peritoneal dialysis patients [20]. Al-Saady et al. showed a hypercoagulable state in patients with chronic renal failure as possible explanation for the increased occurrence of cardiovascular and cerebrovascular events [21]. Hypercoagulation being higher in patients on peritoneal dialysis than haemodialysis patients has been reported by Malyszko et al. [18]. The present study showed a significantly elevated expression of tissue factor on monocytes in haemodialysis patients in comparison to peritoneal dialysis patients. Referring to this, dialysis duration seems to be alleviating since haemodialysis duration was positively correlated with decreased tissue factor expression on monocytes in our study. This may explain that frequent haemodialysis was associated with favourable changes in reduction of left ventricular mass [22]. 
Actually, platelet and monocyte activity markers of atherosclerosis seem to be higher in haemodialysis patients than in peritoneal dialysis patients. In this study, the expression of CD62P on platelets in haemodialysis patients was significantly higher in comparison to that in peritoneal dialysis patients. Scialla et al. evaluated the positive association between soluble P-selectin and atherosclerotic cardiovascular disease in dialysis patients [23]. Especially, male patients showed significantly higher levels of soluble P-selectin and increased cardiovascular disease. Furthermore, this study underlines previous results of Ashman et al. [24] with regard to an increased activation of platelets in dialysis patients, demonstrating more platelet-monocyte aggregates in haemodialysis patients in comparison to those in peritoneal dialysis patients.

Another attempt to reduce cardiovascular disease is to target classical cardiovascular risk factors. In this line, surprisingly, the $4 \mathrm{D}$ and AURORA study showed no beneficial effect for lipid-lowering strategies in diabetic and nondiabetic haemodialysis patients $[25,26]$. In our study, a higher proportion of PD patients received statins. This could be a limitation of our study results, since potential antiinflammatory properties of statins could have attenuated platelet and monocyte activity markers. In contrast to the negative results in clinical trials in haemodialysis patients, simvastatin reduced increased soluble CD40L levels in peritoneal dialysis patients [27]. Expression of CD40L on platelets in this study however did not differ significantly between haemodialysis and peritoneal dialysis patients. Schwabe et al. demonstrated elevated levels of sCD40L in patients with chronic renal disease in comparison to healthy controls [28]. This and other studies raise the question whether dialysis modality may influence cardiovascular risk and mortality [29-31]. In the DOC study, after correction for possible confounders, no statistically significant difference was found in mortality between HD and PD patients [32]. Similarly, no difference was found for the de novo development of coronary heart disease [33]. But there exist potential differences between HD and PD in influencing cardiovascular risk factors, which are altered by uremic toxins [34], fluid overload, and inflammation [35]. Indeed, prior publications are heterogeneous in valuating possible advantages and disadvantages of the two dialysis modalities HD and PD [29-31].

Several toxins such as urea contribute to impaired platelet function and are cleared by dialysis. Serum levels of these dialysable substances still do not correlate with bleeding time or platelet adhesion [36]. The net effect, however, is a reduction of platelet reactivity and an enhanced formation of platelet-dependent fibrin clots contributing to the improved platelet function after dialysis.

We provide evidence that cytokine plasma levels of TNF alpha are significantly higher in haemodialyis patients compared to those in peritoneal dialysis patients, but no significant differences in the other cytokines assessed were detectable.

In peritoneal dialysis, platelet hyperactivity may occur due to common hypoalbuminemia and dyslipidaemia [37]. On the other hand, the higher GPIIb/IIIa expression, a lack of systemic heparinization, an improved clearance of "middle molecules," and the absence of the artificial surfaces of the dialyser and blood tubing account for a lower risk of haemorrhage $[38,39]$. It is known that a disturbed platelet function is an important cause for thromboembolic and haemorrhagic complications in ESRD patients.

Dialysis therapy is the therapeutic standard in patients with ESRD but cardiovascular morbidity and mortality remain unacceptably high in such patients. In the present study, platelet and monocyte activity markers of atherosclerosis seem to be higher in HD patients in comparison to PD patients, possibly suggesting a higher risk of atherosclerotic morbidity and mortality in this major subgroup of dialysis patients.

\section{Conflicts of Interest}

All authors have no conflicts of interests to disclose.

\section{References}

[1] G. Eknoyan, G. J. Beck, A. K. Cheung et al., "Effect of dialysis dose and membrane flux in maintenance hemodialysis," The New England Journal of Medicine, vol. 347, no. 25, pp. 2010 2019, 2002.

[2] R. Paniagua, D. Amato, E. Vonesh et al., "Effects of increased peritoneal clearances on mortality rates in peritoneal dialysis: ADEMEX, a prospective, randomized, controlled trial," Journal of the American Society of Nephrology, vol. 13, no. 5, pp. 1307-1320, 2002.

[3] A. Y. Wang, C. W. Lam, M. Wang et al., "Increased circulating inflammatory proteins predict a worse prognosis with valvular calcification in end-stage renal disease: a prospective cohort study," American Journal of Nephrology, vol. 28, no. 4, pp. 647-653, 2008.

[4] A. Sagripanti and G. Barsotti, "Bleeding and thrombosis in chronic uremia," Nephron, vol. 75, no. 2, pp. 125-139, 1997.

[5] E. P. Evans, R. A. Branch, and A. L. Bloom, "A clinical and experimental study of platelet function in chronic renal failure," Journal of Clinical Pathology, vol. 25, no. 9, pp. 745753, 1972.

[6] M. Gawaz, F. J. Neumann, T. Dickfeld et al., "Activated platelets induce monocyte chemotactic protein-1 secretion and surface expression of intercellular adhesion molecule- 1 on endothelial cells," Circulation, vol. 98, no. 12, pp. 11641171, 1998.

[7] P. Boccardo, G. Remuzzi, and M. Galbusera, "Platelet dysfunction in renal failure," Seminars in Thrombosis and Hemostasis, vol. 30, no. 5, pp. 579-589, 2004.

[8] P. Libby, P. M. Ridker, and A. Maseri, "Inflammation and atherosclerosis," Circulation, vol. 105, no. 9, pp. 1135-1143, 2002.

[9] M. Gawaz, K. Brand, T. Dickfeld et al., "Platelets induce alterations of chemotactic and adhesive properties of endothelial cells mediated through an interleukin-1-dependent mechanism. Implications for atherogenesis," Atherosclerosis, vol. 148, no. 1, pp. 75-85, 2000.

[10] P. S. Frenette, R. C. Johnson, R. O. Hynes, and D. D. Wagner, "Platelets roll on stimulated endothelium in vivo: an interaction mediated by endothelial P-selectin," Proceedings of the National Academy of Sciences of the United States of America, vol. 92, no. 16, pp. 7450-7454, 1995. 
[11] F. Locatelli, L. Del Vecchio, and C. Manzoni, "Morbidity and mortality on maintenance haemodialysis," Nephron, vol. 80, no. 4, pp. 380-400, 1998.

[12] A. E. May, T. Kälsch, S. Massberg, Y. Herouy, R. Schmidt, and M. Gawaz, "Engagement of glycoprotein IIb/IIIa (alpha(IIb)beta3) on platelets upregulates CD40L and triggers CD40L-dependent matrix degradation by endothelial cells," Circulation, vol. 106, no. 16, pp. 2111-2117, 2002.

[13] F. Mach, U. Schönbeck, G. K. Sukhova et al., "Functional CD40 ligand is expressed on human vascular endothelial cells, smooth muscle cells, and macrophages: implications for CD40-CD40 ligand signaling in atherosclerosis," Proceedings of the National Academy of Sciences of the United States of America, vol. 94, no. 5, pp. 1931-1936, 1997.

[14] N. Yorioka, Y. Taniguchi, K. Yamashita et al., "Tissue factor and tissue factor pathway inhibitor in hemodialysis patients," The International Journal of Artificial Organs, vol. 21, no. 11, pp. 699-701, 1998.

[15] R. Pirzer, E. Elmas, D. Haghi et al., "Platelet and monocyte activity markers and mediators of inflammation in Takotsubo cardiomyopathy," Heart and Vessels, vol. 27, no. 2, pp. 186192, 2012.

[16] Y. C. Chuang, J. B. Chen, L. C. Yang, and C. Y. Kuo, "Significance of platelet activation in vascular access survival of haemodialysis patients," Nephrology, Dialysis, Transplantation, vol. 18, no. 5, pp. 947-954, 2003.

[17] J. T. Daugirdas and A. A. Bernardo, "Hemodialysis effect on platelet count and function and hemodialysis-associated thrombocytopenia," Kidney International, vol. 82, no. 2, pp. 147-157, 2012.

[18] J. Malyszko, J. S. Malyszko, and M. Mysliwiec, "Comparison of hemostatic disturbances between patients on CAPD and patients on hemodialysis," Peritoneal Dialysis International, vol. 21, no. 2, pp. 158-165, 2001.

[19] K. Pawlak, D. Pawlak, and M. Mysliwiec, "Tissue factor and urokinase-type plasminogen activator system are related to the presence of cardiovascular disease in hemodialysis patients," Thrombosis Research, vol. 120, no. 6, pp. 871-6, 2007.

[20] K. Pawlak, D. Pawlak, and M. Mysliwiec, "Association between tissue factor, its pathway inhibitor and oxidative stress in peritoneal dialysis patients," Blood Coagulation \& Fibrinolysis, vol. 18, no. 5, pp. 467-471, 2007.

[21] N. M. Al-Saady, E. W. Leatham, S. Gupta, J. T. Kwan, J. B. Eastwood, and C. A. Seymour, "Monocyte expression of tissue factor and adhesion molecules: the link with accelerated coronary artery disease in patients with chronic renal failure," Heart, vol. 81, no. 2, pp. 134-140, 1999.

[22] FHN Trial Group, G. M. Chertow, N. W. Levin et al., "Incenter hemodialysis six times per week versus three times per week," The New England Journal of Medicine, vol. 363, no. 24, pp. 2287-2300, 2010.

[23] J. J. Scialla, L. C. Plantinga, W. H. Kao, B. Jaar, N. R. Powe, and R. S. Parekh, "Soluble P-selectin levels are associated with cardiovascular mortality and sudden cardiac death in male dialysis patients," American Journal of Nephrology, vol. 33, no. 3, pp. 224-230, 2011.

[24] N. Ashman, M. G. Macey, S. L. Fan, U. Azam, and M. M. Yaqoob, "Increased platelet-monocyte aggregates and cardiovascular disease in end-stage renal failure patients," Nephrology, Dialysis, Transplantation, vol. 18, no. 10, pp. 2088-2096, 2003.
[25] V. Krane and C. Wanner, "At which stage of chronic kidney disease should dyslipidemia be treated?" Nature Clinical Practice. Nephrology, vol. 2, no. 4, pp. 176-177, 2006.

[26] B. C. Fellstrom, A. G. Jardine, R. E. Schmieder et al., "Rosuvastatin and cardiovascular events in patients undergoing hemodialysis," The New England Journal of Medicine, vol. 360, no. 14, pp. 1395-1407, 2009.

[27] J. Malyszko, J. S. Malyszko, T. Hryszko, and M. Mysliwiec, "Increased soluble CD40L levels are reduced by long-term simvastatin treatment in peritoneally dialyzed patients," Blood Coagulation \& Fibrinolysis, vol. 15, no. 6, pp. 463-467, 2004.

[28] R. F. Schwabe, H. Engelmann, S. Hess, and H. Fricke, "Soluble CD40 in the serum of healthy donors, patients with chronic renal failure, haemodialysis and chronic ambulatory peritoneal dialysis (CAPD) patients," Clinical and Experimental Immunology, vol. 117, no. 1, pp. 153-158, 1999.

[29] W. Van Biesen, F. Verbeke, and R. Vanholder, "Cardiovascular disease in haemodialysis and peritoneal dialysis: arguments pro peritoneal dialysis," Nephrology, Dialysis, Transplantation, vol. 22 , no. 1 , pp. 53-58, 2007.

[30] S. K. Ganesh, T. Hulbert-Shearon, F. K. Port, K. Eagle, and A. G. Stack, "Mortality differences by dialysis modality among incident ESRD patients with and without coronary artery disease," Journal of the American Society of Nephrology, vol. 14, no. 2, pp. 415-424, 2003.

[31] A. G. Stack, D. A. Molony, N. S. Rahman, A. Dosekun, and B. Murthy, "Impact of dialysis modality on survival of new ESRD patients with congestive heart failure in the United States," Kidney International, vol. 64, no. 3, pp. 1071-1079, 2003.

[32] M. Sanabria, J. Muñoz, C. Trillos et al., "Dialysis outcomes in Colombia (DOC) study: a comparison of patient survival on peritoneal dialysis vs hemodialysis in Colombia," Kidney International. Supplement, vol. 108, pp. S165-S172, 2008.

[33] F. C. Trespalacios, A. J. Taylor, L. Y. Agodoa, G. L. Bakris, and K. C. Abbott, "Heart failure as a cause for hospitalization in chronic dialysis patients," American Journal of Kidney Diseases, vol. 41, no. 6, pp. 1267-1277, 2003.

[34] R. Vanholder, G. Glorieux, N. Lameire, and European Uremic Toxin Work Group, "Uraemic toxins and cardiovascular disease," Nephrology, Dialysis, Transplantation, vol. 18, no. 3, pp. 463-466, 2003.

[35] R. Pecoits-Filho, O. Heimbürger, P. Bárány et al., “Associations between circulating inflammatory markers and residual renal function in CRF patients," American Journal of Kidney Diseases, vol. 41, no. 6, pp. 1212-1218, 2003.

[36] G. Remuzzi, M. Livio, G. Marchiaro, G. Mecca, and G. de Gaetano, "Bleeding in renal failure: altered platelet function in chronic uraemia only partially corrected by haemodialysis," Nephron, vol. 22, no. 4-6, pp. 347-353, 1978.

[37] E. M. Sloand, M. M. Bern, and A. Kaldany, "Effect on platelet function of hypoalbuminemia in peritoneal dialysis," Thrombosis Research, vol. 44, no. 4, pp. 419-425, 1986.

[38] R. M. Lindsay, M. Friesen, F. Koens, A. L. Linton, D. Oreopoulos, and G. de Veber, "Platelet function in patients on long term peritoneal dialysis," Clinical Nephrology, vol. 6, no. 2, pp. 335-339, 1976.

[39] F. Salvati and M. Liani, "Role of platelet surface receptor abnormalities in the bleeding and thrombotic diathesis of uremic patients on hemodialysis and peritoneal dialysis," The International Journal of Artificial Organs, vol. 24, no. 3, pp. 131-135, 2001. 


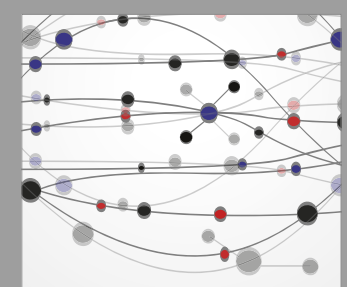

The Scientific World Journal
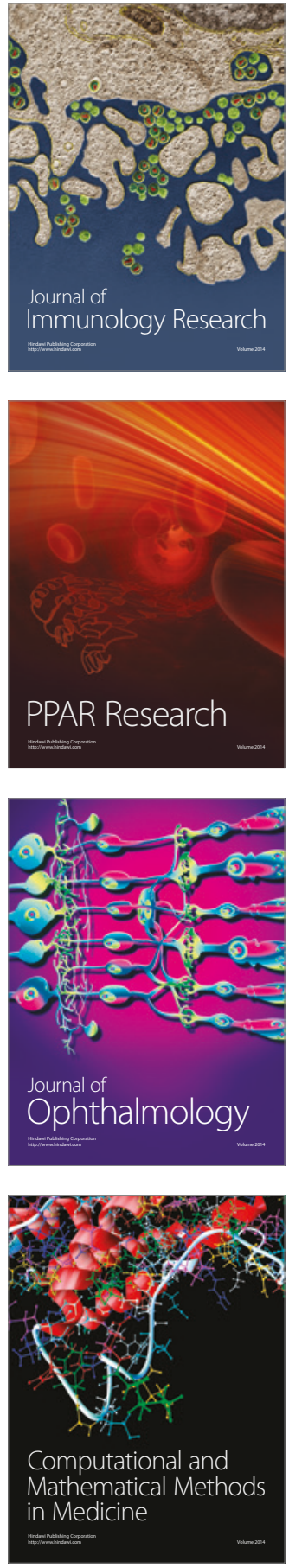

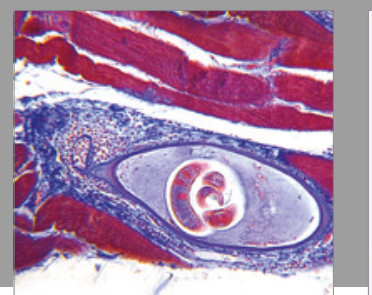

Gastroenterology Research and Practice
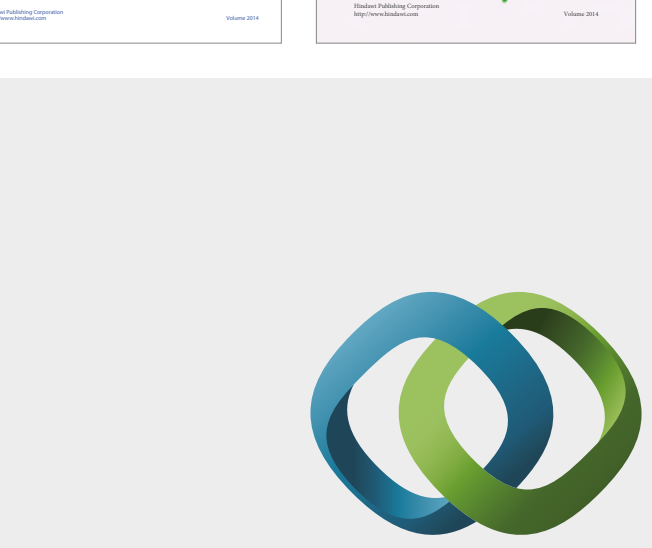

\section{Hindawi}

Submit your manuscripts at

https://www.hindawi.com
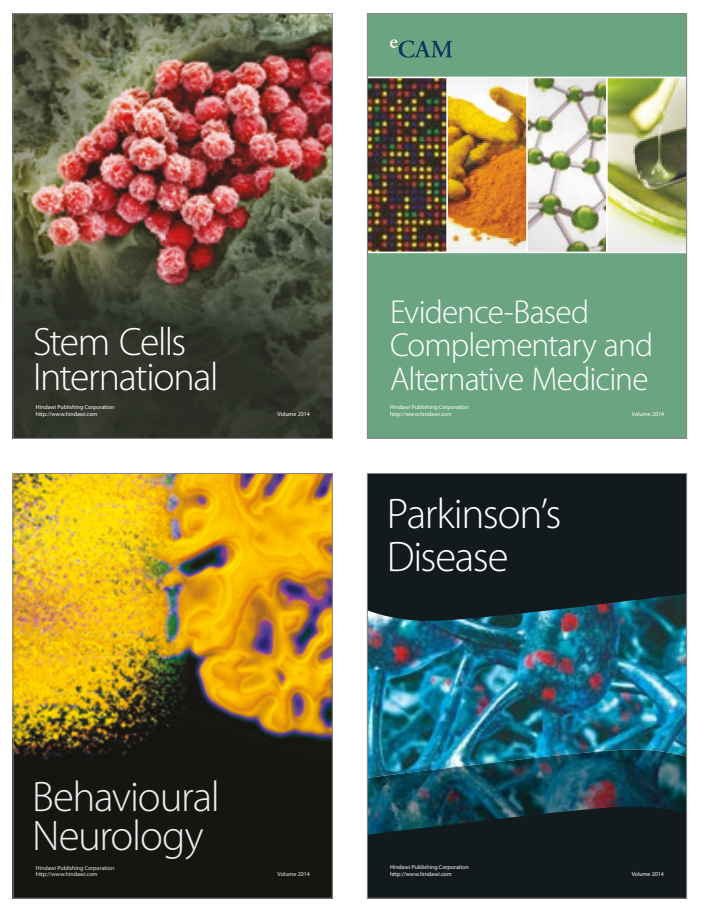
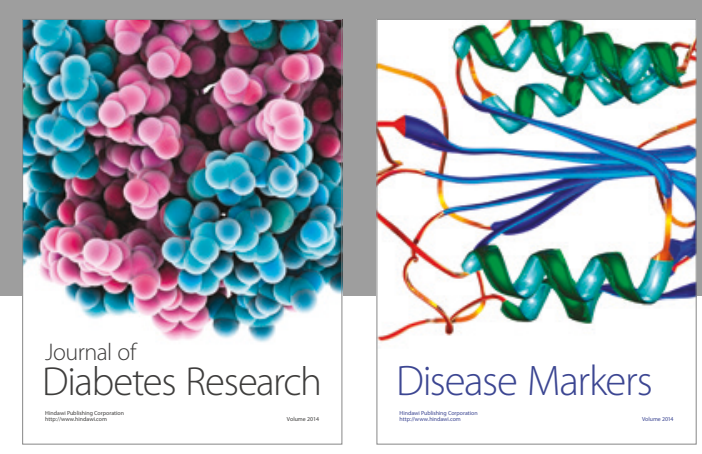

Disease Markers
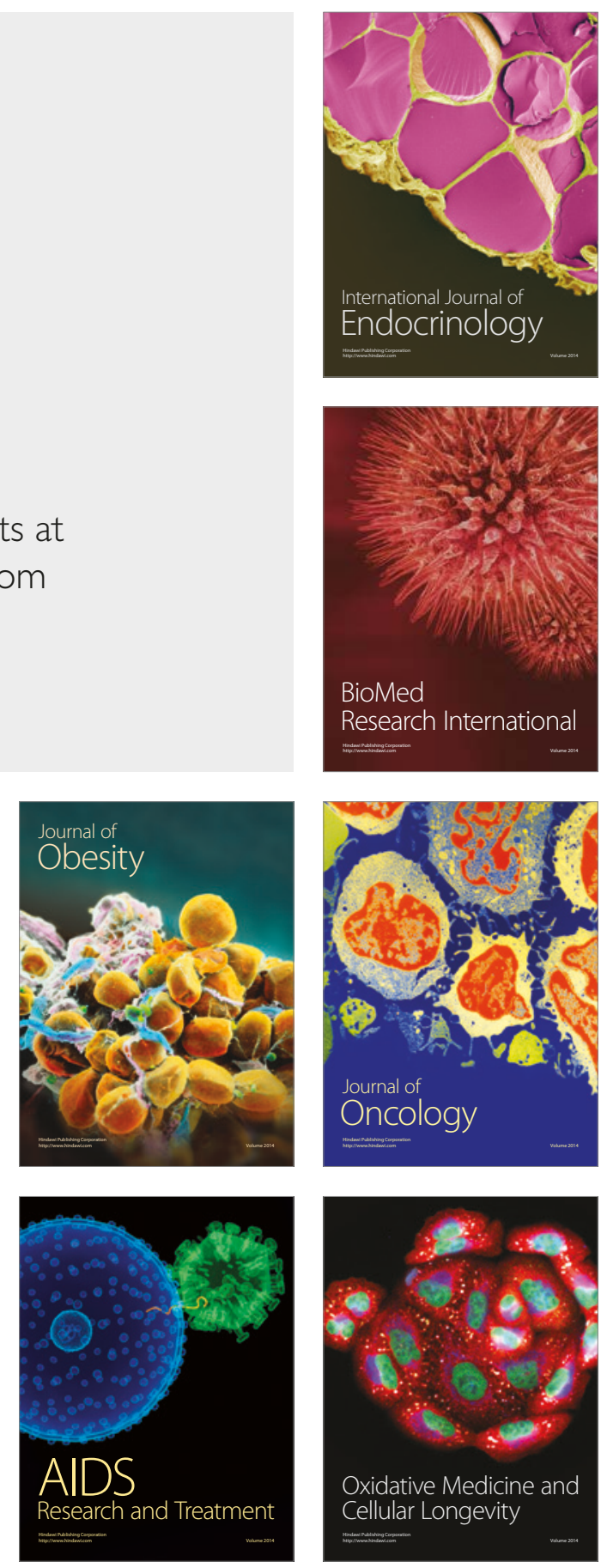